\title{
Zum 65. Geburtstag von Prof. Malte Bühring - Danksagung und Versuch einer Würdigung ${ }^{1}$
}

Zum 65. Geburtstag von Herrn Prof. Dr. med. Malte Bühring am 8.1.2004 sei nachträglich an dieser Stelle herzlich gratuliert. Er gibt Anlass zum Versuch, sein Lebenswerk, insbesondere aber die Zeit als Lehrstuhlinhaber für Naturheilkunde in Berlin zu würdigen. Es soll im Folgenden versucht werden, seine Bedeutung für die Naturheilkunde in jüngerer Zeit nachzuvollziehen und in einen Kontext zu anderen Persönlichkeiten und Entwicklungen zu stellen, um somit ansatzweise die Frage zu beantworten, ob es so etwas wie eine «Ära Bühring» gibt.

Seine 14 Arbeitsjahre in Berlin standen im Dienste von $\mathrm{Pa}-$ tienten, Mitarbeitern, einer klinischen Abteilung unter zwei verschiedenen Trägern, eines Universitäts-Klinikums und des Fachgebiets mit vielen nationalen wie internationalen Kooperationen. Sein Klinikum wechselte 1994 zunächst nur den Namen vom etwas profanen «Klinikum Steglitz» zum «Universitäts-Klinikum Benjamin Franklin» der Freien Universität, fusionierte dann aber im Jahr 2003 mit der Medizinischen Fakultät der Humboldt-Universität zur neuen Charité Universitätsmedizin Berlin. Für die Abteilung für Naturheilkunde bedeutete diese Entwicklung möglicherweise auch den Abschluss eines langen historischen Bogens über das 20. Jhdt.: Während Bührings Dienstzeit als Inhaber des ersten Lehrstuhls für Naturheilkunde nach dem 2. Weltkrieg in Deutschland, dessen Initiierung von der West-Berliner Medizin ausging, kam diese Abteilung unter das grosse Dach der neuen Charité, die in der langen Geschichte ihres Standorts in Berlin-Mitte eine Tradition in diesem Fachgebiet aufweisen kann.

\footnotetext{
${ }^{1}$ Dieser Beitrag ist die geringfügig überarbeitete Rede des Autors auf einer Festveranstaltung zur Emeritierung von Herrn Prof. Dr. med. Malte Bühring am 6. März 2004 in Berlin.
}

\section{Der Berliner Lehrstuhl im Umfeld der Entwicklung} in Medizin und Naturheilkunde

Als die Charité im Jahre 1901 mit der Einrichtung einer Hydrotherapeutischen Anstalt und später auch dem dazugehörigen Lehrstuhl zunächst das akademische Gebiet Hydrotherapie, später daraus hervorgehend auch «Natürliche Heilund Lebensweise» in die akademische Medizin einführte, wobei sie historisch der Wiener Medizinischen Fakultät als ihrem weltweit wohl einzigen ernstzunehmenden Konkurrenten folgte, gehörte eine ärztlich geleitete Naturheilkunde bereits seit etwa 50 Jahren zum festen Bestandteil der medizinischen Versorgung in Deutschland. Sie sollte jedoch erst sehr viel später - in den 80er und 90er Jahren des 20. Jahrhunderts - eine Entwicklung erfahren, die man im Nachhinein zu Recht als einen Boom bezeichnen darf. Die rein quantitative Beschreibung dieser Entwicklung, wie sie sich z.B. in demoskopischen Untersuchungen, in der rasch wachsenden Zahl weitergebildeter Ärzte oder in neu gegründeten Abteilungen und Kliniken festhalten lässt, lässt notwendigerweise die zugrunde liegenden Ursachen im Dunklen. Man sucht sie im Erstarken der ökologischen Bewegung, im Misstrauen gegenüber einer als bedrohlich erlebten «Chemie», in einer neuen Zuwendung zur Natur und in der Gefährdung der Natur durch technischzivilisatorische Prozesse. Die letzten beiden Beweggründe werden vor allem für die Nutzer geltend gemacht. Für viele Ärzte wurde diese am Rande ihres Denkens und Handelns eigentlich ja schon immer vorhandene Disziplin vermutlich erst dann als Betätigungsfeld attraktiv, als die Kritik an der herrschenden Medizin immer breiter formuliert wurde. Hierfür steht Ivan Illichs berühmtes Buch «Die Nemesis der Medizin» stellvertretend für viele andere Ansätze. Gleichzeitig gab der Gesetzgeber spätestens mit der ersten Fassung des Arzneimittelgesetzes von 1977 einen amtlichen Hinweis darauf, dass es «besondere Therapierichtungen» gibt, namentlich die Phytotherapie, die anthroposophische Medizin und die Homöopathie, und diese auch gebührend zu berücksichtigen seien. 
Die Zahl der Ärzte, die sich mit klassischen Naturheilverfahren bzw. mit ausgewählten einzelnen Verfahren wie der Phytotherapie oder angrenzenden Gebieten wie Akupunktur oder Neuraltherapie beschäftigten, stieg in den 80er und 90er Jahren steil auf mehrere Tausend an. Ende 2000 wiesen 10.746 Ärzte die Zusatzbezeichnung «Naturheilverfahren» auf, etwa 50\% der deutschen Ärzte gaben an, zumindest gelegentlich Phytotherapeutika einzusetzen. Polemiken um Berechtigung und Stellenwert dieser Therapierichtungen nahmen ebenfalls an Schärfe zu. Gemessen am heutigen Stand war die klinische Forschung damals rudimentär. Eine akademische Repräsentanz gab es nicht. Es war die Zeit, an der an vielen Universitäten, oft durch Fachschaften initiiert, sog. Ringvorlesungen stattfanden.

Die Forschungsförderung des damaligen Bundesministers für Forschung und Technologie hatte bereits 1983 das Förderprogramm «Unkonventionelle Methoden der Krebsbekämpfung» aufgelegt. Aus heutiger Sicht weisen damals geförderte Vorhaben eklatante Schwächen auf, der Kreis der ärztlichen Antragsteller hatte praktisch keine Erfahrung im Forschungsgeschäft. In der bei Ärzten und in der Öffentlichkeit herrschenden Euphorie über die Entwicklung der Naturheilverfahren in der medizinischen Versorgung gingen diese Mängel an akademischer Infrastruktur zunächst unter.

Betrachtet man den Zeitpunkt der Entwicklung des Berliner Lehrstuhls aus einer anderen Perspektive, so sind hier in den 80er Jahren mehr politische Betätigung und akademische Selbstverwaltung zu beobachten: Die Gesundheitspolitik war bundesweit zu einem immer wichtigeren Instrument geworden. Es gab zwar einerseits schon den Begriff der sog. Kostenexplosion, andererseits befanden wir uns auf einem Wachstumsmarkt mit schier unbegrenzten Möglichkeiten. West-Berlin erlebte den Wandel von einer nur mit ungeheuren Subventionen mühsam am Leben erhaltenen Industrie- zur Dienstleistungsmetropole. 1985, während seiner Dienstzeit als Gesundheitssenator, forderte Ulf Fink, der heute Vorsitzender des Kneipp-Bundes ist und bis vor kurzem auch Vorsitzender der Internationalen Kneippföderation war, Berlin zu einem «Mekka der Medizin» zu machen. Die Förderung der Naturheilverfahren lag ihm schon immer am Herzen, und er gewann einige führende akademische Persönlichkeiten der West-Berliner Medizin für diese Idee. 1988 wurde die Errichtung eines Lehrstuhls für Naturheilkunde in Kooperation mit einer bereits seit 1951 arbeitenden klinischen naturheilkundlichen Abteilung, damals am Krankenhaus Moabit, beschlossen; Malte Bühring wurde am 1.10.1989 zum Lehrstuhlinhaber berufen.

\section{«Evidence-based medicine» als strukturbildendes Element der 90er Jahre}

Für die innermedizinische Entwicklung kann man die 90er Jahre auch als das Jahrzehnt des Siegeszugs des Anspruchs auf «evidence-based medicine» (EBM) charakterisieren. Eine Publikation der Gruppe um David Sackett im «Lancet» im Jahre 1992, die für eine breite medizinische Öffentlichkeit die- ses neue Zeitalter einläutete, erschien, als Prof. Bühring am Lehrstuhl für Naturheilkunde gerade die erste einer geplanten Serie von Konsensus-Konferenzen, zunächst zur sog. Fiebertherapie, durchgeführt und die Ergebnisse publiziert hatte. Damit arbeitete man, ohne sich dessen bewusst zu sein, auf der Ebene IV, also im Erdgeschoss des Sackett'schen EBMGebäudes. Die Frage, wie sich die Naturheilkunde zum Anspruch von EBM verhalten kann und sollte, kann als exemplarisch für andere Felder gelten, auf denen die administrativen, sozialtechnischen und letztlich ökonomischen Regeln in der Medizin festgeschrieben werden. Als Steuerungsinstrumente traten später z.B. Positiv- oder Negativ-Listen, Entscheidungen des Bundesausschusses der Ärzte und Krankenkassen, Leitlinien, neue Vergütungssysteme usw. hinzu. Sehr bald stellte Prof. Bühring die Frage, ob die Naturheilkunde in der zunehmenden Verbürokratisierung der Medizin einen Sonderstatus beanspruchen könne. Woraus liesse er sich ableiten? Aus der überraschend hohen Lebenserwartung der Naturheilkunde, die Wurzeln in einer vorwissenschaftlichen Ära aufweist? Aus ihrer hohen Popularität oder gar ihrer Praktikabilität in einer frei organisierten und frei finanzierten Medizin jenseits der engen Regularien der Kostenträger?

Prof. Bühring wies immer wieder auf Probleme bei der Erfüllung des EBM-Anspruches für die Naturheilkunde hin: Sie wird, gemessen am Stand ihrer klinischen Dokumentation, insbesondere an randomisierten klinischen Studien, lange Zeit nicht mit der konventionellen Medizin standhalten können. Sie muss dies auch nur bedingt, da aufgrund langer und vielfältiger Erfahrungen z.B. ihr Gefährdungspotential für den Patienten niedriger liegt. Sie kann, wenn überhaupt, nur sehr langsam und durch gezielte Förderung das Innovationspotenzial erreichen, das z.B. die medikamentöse Therapie im Zeitalter des «pharma engineering», d.h. der gentechnisch gesicherten Herstellbarkeit nahezu jeden Moleküls, haben muss. Letztlich gilt es auch, einen ganz besonderen Charme der Naturheilkunde zu wahren, der in ihrer emotionalen Nähe und Plausibilität für den Patienten besteht. Dieser stünde auf dem Spiel, wenn jedes sinnlich-empathische Empfinden des Patienten auf einer biochemisch-molekularen Ebene dargestellt werden müsste.

Mittlerweile ist diese Diskussion sehr viel weiter gekommen, und Sacketts Modellvorstellungen haben sich weitgehend durchgesetzt. Sie finden unzweifelhaft ihre Stärke bei Therapieformen, deren individueller Nutzen für Patient wie für Arzt weder vorhersehbar noch unmittelbar nachvollziehbar ist, und die eine hohe Zahl zu Behandelnder, in der Grössenordnung von zehn bis einhundert, für einen einzigen Therapieerfolg voraussetzen. Ein beliebtes Beispiel ist die medikamentöse Therapie von Fettstoffwechselstörungen: Hier kann EBM sicherlich einen guten Wirksamkeitsnachweis liefern und gleichzeitig einen Schutz z.B. gegen Scheininnovationen darstellen.

Es gilt aber auch weiterhin, die Grenzen von EBM aufzuzeigen. In der Naturheilkunde steht die Pyramide des wissen- 
schaftlichen Erkenntnisprozesses sowohl bezüglich der Quantität gesicherten Wissens auf seinen einzelnen Ebenen als auch seiner Richtung «natürlicherweise» auf dem Kopf. Hier möchte und muss die Forschung Grundlagen über Therapien nachliefern, die seit hunderten von Jahren tausend-, wenn nicht millionenfach praktiziert werden, und die nicht einmal nach einer möglichen negativen Bewertung durch den EBMProzess an Popularität einbüssen oder gar von der Bildfläche verschwinden dürften. Insofern stellen sich hier neue Aufgaben in der Bewertung; ein kompromissloses Aufspringen auf den abfahrenden EBM-Express wäre dem Charakter der Naturheilverfahren nicht gerecht geworden und wäre auch mit dem Risiko von Fehleinschätzungen verbunden gewesen. Dies klar gesagt zu haben, ist ein grosses Verdienst von Prof. Bühring.

\section{Neue Entwicklungen}

Während Prof. Bührings Dienstzeit herrschte in den 90er Jahren des letzten Jahrhunderts eine regelrechte Aufbruchsstimmung, zu der es sehr erfreuliche Anlässe gab: z.B. hatte die Gesundheitsministerin Nordrhein-Westfalens, Frau B. Fischer, mit beherztem Engagement landesweit einen Wettbewerb für zwei Modellkliniken mit Naturheilverfahren ausgeschrieben. Herr Bühring erhielt Besuch von mehreren der über 20 Projektgruppen, die sich beteiligten. Jahre später führte dies zum Aufbau zweier Modellkliniken an den Standorten der Gewinner dieses Wettbewerbs: 1997 in Hattingen und 1999 in Essen, wo 2001 ein Neubau für die stationäre Behandlung und 2002 die Ambulanz für Traditionelle Chinesische Medizin eingeweiht wurden.

Nach jahrelangen Vorarbeiten von Dieter Melchart und den Mitarbeitern des damaligen Münchener Modells konnte 1998 die erste Modell-Klinik für Naturheilverfahren in der Rehabilitation in Bad Elster eingeweiht werden. Neue Universitätsambulanzen hatten ihren Betrieb zum Teil schon Jahre zuvor in Heidelberg, Bonn und Freiburg aufgenommen, in München später sogar mit tagesklinischen Plätzen. Allerdings muss man anfügen, dass sich die Universitäten schwer taten, die Forschergruppen in ihre Stellenpläne und die ambulanten Behandlungsmöglichkeiten in ihre Budgets einzubauen. Forschung und Sprechstunden konnten zum Teil nur erfolgen, weil die Karl und Veronica Carstens-, die Erich-Rothenfusseroder die Hektor-Stiftung oft über Jahre einsprangen, wo keine oder nur geringe Mittel aus den universitären Etats oder den Erlösen aus der Gesetzlichen Krankenversicherung bereitstanden.

So entstanden in den 90er Jahren langsam Strukturelemente eines wissenschaftlichen Faches, die nicht nur der Fort- und Weiterbildung dienten, sondern auch der Präsentation und Diskussion wissenschaftlicher Ergebnisse. Als herausragendes Ereignis sei die Münchener Konferenz «Evidence-Based Complementary Medicine» im April 2000 als Start einer internationalen Reihe zu nennen, die in San Francisco 2001, in Boston 2002 und in London 2003 ihre Fortsetzung fand.
Am Ende eines Kräfte zehrenden Prozesses von 1993 bis 1996 wurde die «Europäische Gesellschaft für Klassische Naturheilkunde» (ESCNM, European Society for Classical Natural Medicine) mit Prof. Bühring als erstem Präsident gegründet. Es folgten Konferenzserien bei den spanischen Kollegen der «Asociación Española de Medicinas Naturistas» und die «Bodensee»-Konferenzen in Überlingen. Diese wurden ab 1999 mit einer von Prof. Bühring mitinitiierten äusserst erfolgreichen Summer School für Studenten kombiniert.

Die ESCNM fand mit der deutsch- und englischsprachigen Zeitschrift «Forschende Komplementärmedizin» ein ideales Publikationsmedium vor. Deren erste Ausgabe war 1993 erschienen, Prof. Bühring war seit 1996 in der Schriftleitung tätig. Auf Prof. Bührings Initiative ging die Erweiterung des Titels in «Forschende Komplementärmedizin und Klassische Naturheilkunde» im Jahr 2000 zurück. Die Zeitschrift ist seit 1998 in MEDLINE gelistet, was für naturheilkundliche Zeitschriften immer noch nicht selbstverständlich ist.

\section{Studentische Ausbildung und Fächerkonkurrenz.}

Auch in der Lehre emanzipierte sich die Naturheilkunde zu einem akademischen Fach. Als sie 1993 durch Lernzielkatalog und Prüfungsfragen in den Rang eines freiwilligen Faches im Medizinstudium erhoben wurde, erschien es nur logisch, dem damals einzigen Lehrstuhlinhaber für Naturheilkunde in Deutschland die Kompetenz für die Detailaufgaben zu übertragen. In die Neufassung der Approbationsordnung, die ab Sommersemester 2004 sukzessive nun auch den Rahmen für den klinischen Teil des Medizinstudiums abgibt, wurde als zwölfter und letzter sog. Querschnittsbereich «Rehabilitation, Physikalische Medizin, Naturheilverfahren» aufgenommen. Dies stellt einen der wichtigsten und würdevollsten Abschlusspunkte der Entwicklung der 90er Jahre dar, sind doch nun alle 35 medizinischen Fakultäten verpflichtet, hierfür ein Prüfungssystem zu entwickeln. Allerdings sind sie nur dazu aufgerufen, das darauf vorbereitende Lehrangebot zu unterbreiten.

Zunächst sah es Anfang der 90er Jahre so aus, als würde das Vorbild des damaligen Klinikum Steglitz der Freien Universität Berlin kopiert werden - in Mainz, München, Bonn, Würzburg, Rostock, wo auch immer durch Lehraufträge oder Stiftungsinitiativen ein gewisses Gründungsfieber entstanden war. Heute muss man etwas ernüchtert feststellen, dass kurzfristige, eher auf wenige Jahre angelegte Erwartungen später enttäuscht wurden. Die Einrichtung neuer Lehrstühle für Naturheilkunde brauchte wesentlich länger, obwohl es sich «nur» um Stiftungslehrstühle handelte. Aber auch hier sind zwei wichtige Erfolge zu verbuchen - Frau Prof. Karin Kraft konnte am 15.12.2002 die Professur in Rostock nach annähernd 10-jährigen Bemühungen antreten. Die medizinische Fakultät der Universität Duisburg-Essen berief im März 2004 den Chefarzt der Modellklinik für Naturheilkunde und Integrative Medizin in Essen, Prof. Gustav Dobos, auf den Lehrstuhl für Naturheilkunde. 
Mit der Verankerung der Naturheilverfahren in diesem Querschnittsgebiet hat sich der Gesetzgeber im Bereich der studentischen Ausbildung in zwei wichtigen Fragen festgelegt, die in mehreren Diskussionssträngen der 90er Jahre auftauchen und die Prof. Bühring immer engagiert mitgeprägt hatte. Der erste Strang hinterfragt die Stellung der Naturheilkunde in der gesamten Medizin. Handelt es sich um ein eher eigenständiges oder eben ein sog. Querschnittsfach? Letzteres war die Option des Berliner Lehrstuhls, manchmal zur Enttäuschung innerhalb der Szene, die sich mit der Etablierung der Naturheilkunde auch einen ersten Schritt in Richtung auf ein selbständiges klinisches Fach erhoffte, dem konsequenterweise der zugehörige Facharzt hätte folgen müssen. Der Internist Bühring hatte jedoch eher die Vorteile eines Querschnittsfaches gesehen, nämlich in alle grossen klinischen Fächer fest integriert werden zu können. Dies war für ihn allerdings nur unter der Berücksichtigung und Weiterentwicklung - nie dagegen der Aufgabe - tradierter naturheilkundlicher Konzepte vorstellbar. Als nahe stehendes Fach hat die Psychosomatische Medizin zumindest in Deutschland in den letzten zwanzig Jahren den Weg von einer Spezialdisziplin zu einem Querschnittsfach eingeschlagen - eher zum Vorteil ihres theoretischen Ansatzes wie auch der praktischen Bedeutung in der Versorgungslandschaft.

Der zweite Strang betrifft das nicht immer ungespannte Verhältnis zwischen Naturheilverfahren und Physikalischer Medizin. Prof. Bühring war Präsident der «Deutschen Gesellschaft für Physikalische Medizin und Rehabilitation», als er zum Wintersemester 1989/90 den Berliner Lehrstuhl für Naturheilkunde übernahm. Diese zunächst als völlig zufällig erscheinende Gleichzeitigkeit hat natürlich tiefere Ursachen, die man in der Tradition der nur in Deutschland anzutreffenden Lehrstühle für «Physikalische und Diätetische Therapie» suchen kann, die zu Beginn des 20. Jahrhunderts an vielen medizinischen Fakultäten errichtet wurden. Prof. Bühring hatte nach der Emeritierung seines akademischen Lehrers Prof. Pirlet diesen letzten noch in Frankfurt/Main bestehenden Lehrstuhl kommissarisch geleitet. Gäbe es noch Lehrstühle dieser Art, würde uns jede Animosität zwischen Physikalischer Therapie und Naturheilkunde absurd erscheinen.

Im Curriculum der Bundesärztekammer zur Erlangung der Zusatzbezeichnung «Naturheilverfahren» findet sich etwa ein Drittel nahezu wortgleicher Überschneidungen mit dem der «Physikalischen Therapie». In den Katalog zum 1992 eingerichteten Facharzt für Physikalische und Rehabilitative Medizin wurden vice versa auch die Naturheilverfahren - wenngleich ohne jede Erklärung - mit aufgenommen. Die neue Approbationsordnung hat mit den sicherlich sehr knappen Formulierungen eines Gesetzestextes nunmehr ganz klar Nähe und Gleichwertigkeit festgeschrieben. Der neue Querschnittsbereich stellt eine deutliche Aufforderung zum gleichberechtigten Dialog dar.

Am Ende einer «Ära Bühring» sind für das Fachgebiet Naturheilkunde einige klare Positionsgewinne zu verzeichnen.
Wichtige strukturbildende Elemente wie Kliniken, akademische Einrichtungen, insbesondere zwei neue Stiftungsprofessuren, Fachgesellschaften und Zeitschriften sind auf den Weg gebracht worden.

Was wurde nicht erreicht? Hier ist in erster Linie das Ausbleiben öffentlicher Forschungsförderung zu nennen. Die zeitlich sich überlappenden staatlichen Förderungsprogramme UMK (Unkonventionelle Methoden der Krebsbekämpfung) und UMR liefen insgesamt 23 Jahre lang (1982-2005); 2005 laufen die letzten Projekte aus. Im Schnitt wurden ca. $600.000 €$ pro Jahr ausgeschüttet. Trotz vieler Bemühungen wird es auch für erfolgreiche Projekte definitiv keine Möglichkeiten der Anschlussförderung geben. Auch ein entsprechendes Programm der Europäischen Union ist noch nicht in Sicht.

In den USA begann dagegen erst 1992 die staatliche Forschungsförderung für «CAM» mit der Etablierung des «Office of Alternative Medicine at the National Institute of Health» und einem ersten Jahresbudget von 2 Mio. US-\$. Die Nachfolgeinstitution «National Centre for Complementary and Alternative Medicine» (NCCAM, seit 1998) wies 2003 ein Budget von 114,1 Mio. US-\$ auf, zusammen mit Förderungen aus anderen Programmen des NIH ergibt sich ein Fördervolumen durch die US-Bundesregierung in Höhe von etwa 220 Mio. US-\$.

Als zweiter grosser Problembereich muss die öffentliche Regulierung der Krankenversorgung, insbesondere das seit Anfang 2004 geltende Gesundheitsmodernisierungsgesetz mit seiner fatalen Neuformulierung des $\S 34$ genannt werden, dessen Tragweite noch nicht abschätzbar ist.

Die Naturheilkunde muss sich weiterhin einer Vielzahl oft schwer verständlicher Entwicklungen stellen, für die auch die viel zitierte Globalisierung verantwortlich ist. Sie muss dabei jedoch weiterhin überzeugt sein, dass sich auch in der Medizin das Sinnvolle und moralisch Berechtigte letztlich durchsetzen wird.

Ein Beispiel für die Folgen der Globalisierung sei genannt: Die Ankündigung der Zulassung eines neuen Darmkrebsmedikamentes in den USA im März 2004 stellt dem Entwickler und Hersteller weltweit eine Umsatzsteigerung von $2 \mathrm{Mrd}$. US-\$ in Aussicht, lässt seine Kurse steigen und stärkt seine Marktposition auch in Deutschland. Die Gesetzliche Krankenversicherung in Deutschland wird einen grossen Teil zu diesen 2 Mrd. beitragen (müssen?), woraus sich bei stagnierenden oder gar fallenden Gesamtbudgets sofort die Frage nach weiteren Einsparpotenzialen ergibt. Die Erstattungsfähigkeit von Therapien aus der Naturheilkunde und angrenzenden Methoden stellte in letzter Zeit einen der Puffer dar, die für die Einsparungen herhalten müssen. Wie ist dies trotz ihrer Popularität möglich? Gewinner und Verlierer in solchen Verteilungskämpfen zeichnen sich beinahe naturgemäss durch unterschiedliche, wenn nicht diametrale Eigenschaften aus. Die Naturheilkunde kostet eher wenig, verfügt derzeit über ein geringes Innovationspotential und ist weder auf der Anbieter- noch auf der Verbraucherseite national wie internatio- 
nal gut organisiert. Der Zynismus der gegenwärtigen gesundheitspolitischen Diskussion kommt darin zum Ausdruck, dass die Bundesregierung mit der weitgehenden Streichung rezeptfreier Präparate aus der GKV-Erstattung im Unterschied zu früheren Situationen diesmal überhaupt nicht deren Wirksamkeit anzweifeln möchte - der Patient möge sie sich bei seinem durchaus berechtigten Bedarf nur bitte selber kaufen!

Was sind die wichtigsten Aufgaben für die Zukunft?

Für wichtige Indikationen müssen die zahlreichen naturheilkundlichen und komplementärmedizinischen Verfahren bewertet werden. Es müssen randomisierte Studien zum Vergleich mit Standardtherapien, aber auch innerhalb des Fachgebietes durchgeführt werden.

In Langzeit-Verlaufsstudien, die nicht randomisiert sein müssen, gilt es aufzuzeigen, dass chronisch Kranke in einer integrierten naturheilkundlichen Langzeitversorgung, im viel zitierten «Setting», bessere Zufriedenheit und bessere objektive Krankheitsverläufe erzielen.

Die Zusammenarbeit und Solidarität der Gruppen und der oft nach Einzelmethoden aufgesplitterten Fachgesellschaften muss besser werden. Derzeit ist die Naturheilkunde mit keiner Stimme in der «Arbeitsgemeinschaft Wissenschaftlich-
Medizinischer Fachgesellschaften» (AWMF) vertreten, an die sich z.B. die Bundesregierung bei Beratungsbedarf wendet. Neue Kooperationsansätze wie die Arbeit des «FORUM naturheilkundlicher und komplementärmedizinischer universitärer Gruppen» oder die Gründung der Fachgesellschaft «Deutsche Gesellschaft für Naturheilkunde e.V.» aus der Europäischen Gesellschaft für Klassische Naturheilkunde, an deren Arbeit Prof. Bühring wesentlich beteiligt war, geben Anlass zu Hoffnung.

Wir wünschen Herrn Prof. Bühring Freude und Gelassenheit bei der Beobachtung der Projekte, die in seiner Ära auf den Weg gebracht wurden, und vor allem natürlich gute Gesundheit - ad multos annos.

\section{R. Stange, Berlin}

\section{Literatur}

1 Stange R: Zum 60. Geburtstag von Professor M. Bühring. Forsch Komplementärmed 1999;6:127-128.

2 Stange R (Hrsg.): Naturheilkunde am Ende des 20. Jahrhunderts. Rückblick Gegenwart - Perspektiven. Stuttgart, Hippokrates, 2000.

3 Kraft K: Zum 65. Geburtstag von Prof. Dr. med. Malte Bühring. Forsch Komplementärmed Klass Naturheilkd 2004;11:177. 